\title{
SURVIVAL MODELS IN THE CONTEXT OF MULTIPLICATIVE AND ADDITIVE HAZARDS
}

\author{
Jaqueline Aparecida RAMINELLI ${ }^{1}$ \\ Suely Ruiz GIOLO ${ }^{2}$
}

- ABSTRACT: In survival analysis, multiplicative and additive hazards models provide the two principal frameworks to study the association between the hazard and covariates. When these models are considered for analyzing a given survival dataset, it becomes relevant to evaluate the overall goodness-of-fit and how well each model can predict those subjects who subsequently will or will not experience the event. In this paper, this evaluation is based on a graphical representation of the Cox-Snell residuals and also on a time-dependent version of the area under the receiver operating characteristic (ROC) curve, denoted by $\mathrm{AUC}(t)$. A simulation study is carried out to evaluate the performance of the $\mathrm{AUC}(\mathrm{t})$ as a tool for comparing the predictive accuracy of survival models. A dataset from the Mayo Clinic trial in primary biliary cirrhosis (PBC) of the liver is also considered to illustrate the usefulness of these tools to compare survival models formulated under distinct hazards frameworks.

- KEYWORDS: Model comparison; predictive accuracy; ROC curve; time to event data.

\section{Introduction}

Data involving time to the occurrence of a certain event has been usually referred to as survival data. A complication in analyzing such data is that they are usually censored, meaning that the event time of interest is not fully observed on all subjects under study.

The most well-known regression model proposed for dealing with survival data is the Cox model (COX, 1972), whose validity relies on the assumption of

\footnotetext{
${ }^{1}$ Universidade Estadual de Londrina - UEL, Departamento de Estatística, CEP: 86057-970, Londrina, PR, Brasil. E-mail: raminelli@uel.br

${ }^{2}$ Universidade Federal do Paraná - UFPR, Departamento de Estatística, CEP: 81531-980, Curitiba, PR, Brazil. E-mail: giolo@ufpr.br
} 
proportional hazards. As this assumption may not always be true (for example, the effect of a treatment may change over time), alternative models which allow non-proportional hazards (i.e., time-varying covariate effects) have been proposed.

One of a such model is the Aalen's additive model (AALEN, 1980, 1989). It is a flexible nonparametric model whose coefficients are functions of time without any particular form or dependence on other parameter functions. Since the effect of some covariates may change with time while the effect of others may not depend on time, McKeague and Sasieni (1994) have suggested a submodel of the Aalen's model named semiparametric additive hazards model. It allows some covariates having time-varying effect and others do not. For these two models the covariate effects are modeled on an additive scale and the estimators are given on explicit form.

Other models that can accommodate time-varying covariate effects are a natural extension of the multiplicative Cox model and its semiparametric version (given that the flexibility of the extended Cox model may not be needed for all covariates). For these models, which have been investigated by several authors (e.g., SCHEIKE, 2002; MARTINUSSEN; SCHEIKE, 2006), the covariate effects are modeled on a multiplicative scale.

Since for a specific survival dataset it is not clear in advance which of the models mentioned would be the best, it becomes relevant to assess the goodness-offit and the predictive accuracy for each of them. For that, Abadi et al. (2011) have noted that there are several statistical challenges. One of them is the impossibility of using statistical tests such as the likelihood ratio test, score test and Wald test since the models are not nested (except in special cases). Another is that the likelihood function is difficult to specify for additive hazards models containing nonparametric terms, which implies that likelihood based model selection criteria, such as Akaike's information criterion (AIC), Bayesian information criterion (BIC) and Schwarz's Bayesian criterion (SBC) can not be used in this situation (HUFFER; McKEAGUE, 1991).

With these statistical challenges in mind, the purpose of this paper was to consider a time-dependent version of the area under the receiver operating characteristic (ROC) curve, discussed by Heagerty and Zheng (2005) in the setting of survival models and denoted by $\operatorname{AUC}(t)$, as a procedure to assess the predictive accuracy of hazards models formulated under distinct frameworks (additive and multiplicative). The $\mathrm{AUC}(t)$ allows both the event status and marker value change over time. A graphical representation of the Cox-Snell residuals was also considered for assessing the overall goodness-of-fit of these hazards models.

The paper is organized as follows. In Section 2 we outline the multiplicative and additive hazards models, as well as the Cox-Snell residuals and the timedependent version of the area under the ROC curve for these models. A simulation study is presented in Section 3 and a lifetime dataset is analyzed in Section 4 to illustrate the procedures considered for assessing the overall goodness-of-fit and the predictive accuracy of the models. A discussion ends the paper in Section 5.

Rev. Bras. Biom., Lavras, v.37, n.3, p.306-323, 2019 - doi: 10.28951/rbb.v37i3.391 


\section{Hazards models and related measures}

Let $Y_{i}$ denote the time of an event for individual $i(i=1, \ldots, n)$ and define the observed event time $T_{i}=\min \left(Y_{i}, C_{i}\right)$, where $C_{i}$ is a censoring time. Thus, when $T_{i}=Y_{i}$, the observed time corresponds to an event $\left(\delta_{i}=1\right)$, otherwise the event time is censored at $C_{i}\left(\delta_{i}=0\right)$. For a sample of size $n$, survival data usually contains the realizations of the random variables $\left\{\left(T_{i}, \delta_{i}, X_{i 1}, \ldots, X_{i p}\right)\right\}, i=1, \ldots, n$, where $\delta_{i}$ is the censoring indicator taking value 1 if an event occurs and 0 otherwise, and $X_{i 1}, \ldots, X_{i p}$ are the covariates.

\subsection{Multiplicative hazards models}

The multiplicative hazards models assume that the covariate effects act multiplicatively on some unknown baseline hazard rate. The most well-known multiplicative model was proposed by Cox (1972), whose validity relies on the assumption of proportional hazards. It relates covariates to the hazard function as follows

$$
\lambda\left(t \mid \mathbf{x}_{i}\right)=\lambda_{0}(t) \exp \left(\mathbf{x}_{i}^{T} \boldsymbol{\beta}\right)
$$

where $\lambda_{0}(t)$ is an unspecified baseline hazard function, $\boldsymbol{\beta}$ is a vector of regression coefficients, and $\mathbf{x}_{i}$ is a vector of covariates for the $i$-th individual. Estimation of $\boldsymbol{\beta}$ in model (1) is based on a partial likelihood (Cox, 1975).

Since the assumption of proportional hazards may not hold and might also exist covariates that vary over time, a very flexible extension was proposed for model (1) to allow both time-varying covariates and time-varying covariate effects (ZUCKER; KARR, 1990; MARTINUSSEN et al., 2002; CAI; SUN, 2003). For this extended Cox's model, the hazard function is expressed as

$$
\lambda\left(t \mid \mathbf{x}_{i}(t)\right)=\lambda_{0}(t) \exp \left[\mathbf{x}_{i}^{T}(t) \boldsymbol{\beta}(t)\right],
$$

where $\mathbf{x}_{i}(t)=\left(x_{i 1}(t), \ldots, x_{i p}(t)\right)$ is a vector of time-varying covariates for the $i$-th individual and $\boldsymbol{\beta}(t)=\left(\beta_{1}(t), \ldots, \beta_{p}(t)\right)$ is a $p$-dimensional time-varying regression coefficients. Despite the notation $\mathbf{x}_{i}(t)$, it is worth mentioning that not all covariates need to be time-dependent (e.g., gender and race). Estimation of $\boldsymbol{\beta}(t)$ in model (2) is not straightforward as the estimation of $\boldsymbol{\beta}$ in model (1). For a such purpose, Zucker and Karr (1990) have studied a penalized partial likelihood approach while Murphy and Sen (1991) a sieve approach. Martinussen et al. (2002) have also proposed one-step estimation of the cumulative parameter function $B_{k}(t)=\int_{0}^{t} \beta_{k}(u) d u$. More recently, a global partial likelihood method was proposed by Chen et al. (2012).

Given that the flexibility of the extended Cox model may not be needed for all covariates, it was proposed a submodel of model (2) which allow some covariates with time-varying effect and others do not. The hazard function of this submodel is expressed as

$$
\lambda\left(t \mid \mathbf{x}_{i}(t), \mathbf{z}_{i}(t)\right)=\lambda_{0}(t) \exp \left[\mathbf{x}_{i}^{T}(t) \boldsymbol{\beta}(t)+\mathbf{z}_{i}^{T}(t) \boldsymbol{\gamma}\right]
$$


where $\mathbf{x}_{i}(t)$ and $\mathbf{z}_{i}(t)$ are covariate vectors of dimensions $p$ and $q$, respectively, $\boldsymbol{\beta}(t)$ is a $p$-dimensional regression functions and $\gamma$ is a $q$-dimensional regression vector. Similar to model (2), some or all covariates of $\mathbf{x}_{i}(t)$ and $\mathbf{z}_{i}(t)$ may do not change over time or can be assumed to be fixed at the start of the study. A procedure for estimating the components of $\boldsymbol{\beta}(t)$ and $\boldsymbol{\gamma}$ has been proposed by Martinussen et al. (2002) who also have derived the asymptotic distribution of the estimators.

\section{$2.2 \quad$ Additive hazards models}

An alternative framework in modeling hazard functions is the additive models. For them, the hazard of a covariate is an additive increment on the baseline hazard. The most known nonparametric additive hazards model was proposed by Aalen (1980, 1989). A submodel of the Aalen's model has subsequently been proposed by McKeague and Sasieni (1994) to allow some covariates with time-varying effect and others do not. The hazard functions of these models have, respectively, the form

and

$$
\begin{aligned}
& \lambda\left(t \mid \mathbf{x}_{i}(t)\right)=\lambda_{0}(t)+\mathbf{x}_{i}^{T}(t) \boldsymbol{\beta}(t) \\
& \lambda\left(t \mid \mathbf{x}_{i}(t), \mathbf{z}_{i}(t)\right)=\lambda_{0}(t)+\mathbf{x}_{i}^{T}(t) \boldsymbol{\beta}(t)+\mathbf{z}_{i}^{T}(t) \boldsymbol{\gamma}
\end{aligned}
$$

where $\mathbf{x}_{i}(t), \mathbf{z}_{i}(t), \boldsymbol{\beta}(t)$ and $\boldsymbol{\gamma}$ are defined as in models (2) and (3).

Model (4) is referred to as nonparametric because no assumption is made about the functional forms of $\boldsymbol{\beta}(t)$. Estimation of $\boldsymbol{\beta}(t)$ is based on a least-squares technique (HUFFER; McKEAGUE, 1991) and since direct estimation of $\beta_{k}(t)$ is difficult, it is usual to estimate the cumulative regression functions $B_{k}(t)=\int_{0}^{t} \beta(u) d u, k=$ $1, \ldots, p$. A crude estimate of $\beta_{k}(t)$ is given by the slope of the estimate of $B_{k}(t)$. However, better estimates can be obtained by using kernel smoothing methods (HASTIE et al., 2009). For model (5), estimation of $\boldsymbol{\beta}(t)$ and $\boldsymbol{\gamma}$ is also based on a least-squares technique and kernel smoother (McKEAGUE; SASIENI, 1994).

Although models (2) to (5) allow time-varying covariate effects, it is worth mentioning that the estimation is restricted to a time interval $(0, \tau]$ where $\tau$ is the maximum time $t$ at which the design matrix $\mathbf{X}(t)$ is non-singular.

\subsection{Overall goodness-of-fit and predictive accuracy of the models}

When one of the mentioned hazards models is considered to analyze a survival dataset, it is important to evaluate whether it actually fits the data. For model (1) several graphical and numerical methods based on different residuals (Schoenfeld, Cox-Snell, martingale and deviance residuals) have been proposed for judging its goodness-of-fit (COX; SNELL, 1968; SCHOENFELD, 1982; LIN et al., 1993; GRAMBSCH; THERNEAU, 1994). For models (2) to (5), some techniques based on martingale and cumulative martingale residuals have also been proposed to validate the fit of them (MARTINUSSEN; SCHEIKE, 2006).

Rev. Bras. Biom., Lavras, v.37, n.3, p.306-323, 2019 - doi: 10.28951/rbb.v37i3.391 
In order to evaluate the overall goodness-of-fit of the hazards models described previously, we have focused in this paper on a graphical representation of the CoxSnell residuals $e_{i}, i=1, \ldots, n$, defined for models (1) to (5) as

$$
e_{i}=\left\{\begin{array}{lc}
\widehat{\Lambda}_{0}\left(t_{i}\right) \exp \left(\mathbf{x}_{i}^{T} \widehat{\boldsymbol{\beta}}\right) & \text { model (1) } \\
\int_{0}^{t_{i}} \widehat{\lambda}_{0}(s) \exp \left[\mathbf{x}_{i}^{T}(s) \widehat{\boldsymbol{\beta}}(s)\right] d s & \operatorname{model}(2) \\
\int_{0}^{t_{i}} \widehat{\lambda}_{0}(s) \exp \left[\mathbf{x}_{i}^{T}(s) \widehat{\boldsymbol{\beta}}(s)+\mathbf{z}_{i}^{T}(s) \widehat{\boldsymbol{\gamma}}\right] d s & \operatorname{model}(3) \\
\int_{0}^{t_{i}}\left[\widehat{\beta}_{0}(s)+\mathbf{x}_{i}^{T}(s) \widehat{\boldsymbol{\beta}}(s)\right] d s & \text { model (4) } \\
\int_{0}^{t_{i}}\left[\widehat{\beta}_{0}(s)+\mathbf{x}_{i}^{T}(s) \widehat{\boldsymbol{\beta}}(s)+\mathbf{z}_{i}^{T}(s) \widehat{\gamma}\right] d s & \text { model (5) }
\end{array}\right.
$$

where $\widehat{\Lambda}_{0}(t)$ is the estimated cumulative baseline hazard function. If the model fits the data well, a plot of the Cox-Snell residuals $e_{i}$ against the estimated cumulative hazard rate of $e_{i}$, denoted by $\widehat{\Lambda}\left(e_{i}\right)$, should be roughly a straight line through the origin with slope 1 (LAWLESS, 2002).

Another issue that is important to taken into account when hazards models under different frameworks are considered to analyze a survival dataset is which one has the best predictive acuracy. Considering the comparison of models, Torner (2004) has fitted the standard Cox's model (1) and the Aalen's model (4) to breast cancer data and then performed an informal comparison between them. A similar comparison between models (1) and (4) has also been made by Cao (2005) using laryngeal cancer data and by Abadi et al. (2011) using breast cancer data. The conclusion was that the two models give different pieces of information and should be viewed as complementary methods.

Since only an informal comparison of models has been made by the mentioned authors, would be useful to consider a measure that make feasible to evaluate the predictive accuracy of hazards models formulated under additive or multiplicative frameworks. In this paper, the time-dependent version of the area under the ROC curve was considered for this purpose. To obtain a such area, let $\delta_{i}(t)$ be the event status at time $t$, taking values 1 or 0 , and $M_{i}(t), i=1, \ldots, n$, a longitudinal marker used as a prediction of failure at time $t$. This marker may also denote a risk score computed from a regression or some other model. As usual, it will be assumed that a higher marker value is more indicative of an event. Hence, for a given threshold $c$, the time-dependent sensitivity and specificity can be defined respectively by

$$
\left\{\begin{array}{l}
S e(c, t)=P\left(M_{i}(t)>c \mid \delta_{i}(t)=1\right) \\
S p(c, t)=P\left(M_{i}(t) \leq c \mid \delta_{i}(t)=0\right) .
\end{array}\right.
$$

Since sensitivity and specificity are time-dependent functions, we can define the corresponding ROC curve for any fixed time $t$ as $\operatorname{ROC}(t)$ which plots $1-S p(c, t)$ against $S e(c, t)$ for thresholds $c$. The time-dependent AUC is then defined as

$$
A U C(t)=\int_{-\infty}^{\infty} S e(c, t) d[1-S p(c, t)]
$$


where $[1-S p(c, t)]=\frac{\partial[1-S p(c, t)}{\partial c} d c$.

Several methods have been proposed for estimating the time-dependent ROC curve for censored event times (BLANCHE et al., 2013; SHEN et al., 2015, amongst others). Recently, Kamarudin et al. (2017) presented a review of the current methods which use single or longitudinal marker measurements. In this paper, the cumulative/dynamic (C/D) definition given by Heagerty and Zheng (2000), which has commonly been used in clinical applications, was considered for estimating the time-dependent sensitivity and specificity. For C/D definition, a case is defined as any individual experiencing the event between $t=0$ and time $t$ and a control as an individual remaining event-free at time $t$. Hence, each individual may be a control at the earlier time (when the event time is greater than the target time, i.e. $T_{i}>t$ ) but then contributes as a case for later times (when the event time is less than or equal to the target time, i.e. $T_{i} \leq t$ ).

Thus, the cumulative sensitivity at time $t$ (which corresponds to the probability that an individual has a marker value greater than $c$ among the individuals who experienced the event before time $t$ ), the dynamic specificity at time $t$ (which corresponds to the probability that an individual has a marker value less than or equal to $c$ among those event-free individuals beyond time $t$ ), and the $\operatorname{AUC}(t)$, corresponding to the probability of an individual who experienced the event having a higher marker value than a healthy individual, can be defined as

$$
\begin{aligned}
S e(c, t) & =P\left(M_{i}(t)>c \mid T_{i} \leq t\right) \\
S p(c, t) & =P\left(M_{i}(t) \leq c \mid T_{i}>t\right) \\
A U C(t) & =P\left(M_{i}(t)>M_{j}(t) \mid T_{i} \leq t, T_{j}>t\right), \quad i \neq j .
\end{aligned}
$$

Since the values of $S e(c, t)$ and $S p(c, t)$ fall in the interval [0,1], the marker $M_{i}(t), i=1, \ldots, n$, will be perfect at discriminating between failure and censorship when both take the value 1 . Consequently, the $\operatorname{AUC}(t)$ can be used to characterize the ability of the marker to perform this discrimination over time. The closest is the $\operatorname{AUC}(t)$ of 1 , better is the discriminating ability of the marker at time $t$.

In order to consider the predictive accuracy of the Cox model (1) using the $\operatorname{AUC}(t)$ in the case where the proportional hazards assumption holds, Heagerty and Zheng (2005) have proposed the linear predictor $\mathbf{x}_{i}^{T} \boldsymbol{\beta}$ as the model score $M_{i}(t), i=$ $1, \ldots, n$. Nevertheless, if the proportional hazards is violated, the score they have proposed was $M_{i}(t)=\mathbf{x}_{i}^{T} \boldsymbol{\beta}(t)$, given that a more general model such as model (2) would be more appropriate in this case.

Based on the Heagerty and Zheng proposal, the scores $M_{i}(t), i=1, \ldots, n$, proposed in this paper to assess the predictive accuracy at time $t$ of models (1) to (5) by means of the $\mathrm{AUC}(t)$ are given as follows

$$
M_{i}(t)= \begin{cases}\mathbf{x}_{i}^{T} \boldsymbol{\beta} & \text { model (1) } \\ \mathbf{x}_{i}^{T}(t) \mathbf{B}(t) & \text { models (2) and (4) } \\ \mathbf{x}_{i}^{T}(t) \mathbf{B}(t)+\mathbf{z}_{i}^{T}(t) \boldsymbol{\gamma} t & \text { models (3) and (5), }\end{cases}
$$

Rev. Bras. Biom., Lavras, v.37, n.3, p.306-323, 2019 - doi: 10.28951/rbb.v37i3.391 
where $\boldsymbol{\beta}=\left(\beta_{1}, \ldots, \beta_{p}\right), \mathbf{B}(t)=\left(B_{1}(t), \ldots, B_{p}(t)\right)$, with $B_{k}(t)=\int_{0}^{t} \beta_{k}(u) d u$ for $k=1, \ldots, p$, and $\gamma=\left(\gamma_{1}, \ldots, \gamma_{q}\right)$.

For each specified time $t$, the nearest neighbor estimation (NNE) method (AKRITAS, 1994; HEAGERTY et al., 2000) was used to estimate the ROC curve and its corresponding $\mathrm{AUC}(t)$. The survivalROC package for the $\mathrm{R}$ software $(\mathrm{R}$ CORE TEAM, 2017) helped in this estimation process, and the survival and timereg packages in the estimation of the parameters $\boldsymbol{\beta}, \boldsymbol{\gamma}$, and $\mathbf{B}(t)$ of models (1) to (5).

\section{Simulation study}

A simulation study was carried out in order to evaluate the $\operatorname{AUC}(t)$ as a tool to compare multiplicative and addditive survival models relative to their predictive accuracy. For this study, we have generated survival times $T_{i}(i=1, \ldots, n)$ from the accelerated failure time model (LAWLESS, 2002) considering the Weibull and a continuous covariate $X_{1}$ with time-invariant effect, as well as the lognormal and a continuous covariate $X_{1}$ with time-varying effect, like those shown in Figure 1. The covariate values $\left(x_{1 i}, i=1, \ldots, n\right)$ were generated from an exponential distribution. The sample sizes considered were $n=100,300$ and 500 with $0 \%, 20 \%$ and $40 \%$ of right censoring. Readers interested in survival data-generating processes can see Austin (2012) and Bender et al. (2005), amongst others.

(a)

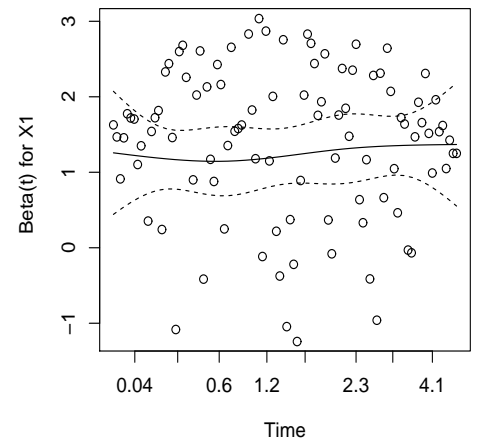

(b)

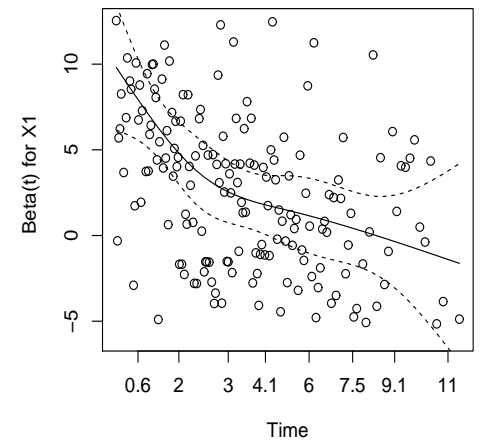

Figure 1 - (a) Covariate $\mathrm{X}_{1}$ with time-invariant effect and (b) with time-varying effect.

Based on 1,000 simulation runs for each combination of $n$ and percentage of censorship, the mean AUC and its corresponding standard error were obtained at several times $t$ for each model. Table 1 shows the simulation results for those scenarios where the continuous covariate $X_{1}$ was considered in the data-generating process with time-invariant effect (the maximum time ranged from 6 to 8 ).

From Table 1, it can be noted that the models presented similar AUC $(t)$ values, as well as that for the extended Cox and Aalen models it was not possible to obtain $\operatorname{AUC}(t)$ for $t \geq 4.5$ when $n=100$ and $t \geq 5$ when $n=300$ and 500. This is due to the estimation procedure for these models to be possible only while the 
design matrix $\mathbf{X}(t)$ is non-singular. Other facts that can be observed from Table 1 are: $i)$ the standard errors decreased as the sample size increased; $i i)$ the standard errors increased as the percentage of censorship increased; iii) the $\operatorname{AUC}(t)$ values decreased as time increased (which is expected due to the reduction of subjects at risk over time); and $i v$ ) a more pronounced decrease in the $\operatorname{AUC}(t)$ values over time was recorded for those scenarios with $20 \%$ and $40 \%$ of censorship than for those with no censorship. Thus, regarding the predictive accuracy of the models, the simulation results indicated that there is no substantial gain in adjusting models with time-varying covariate effects when the effects are time-invariant.

Table 1 - AUC $(t)$ and standard error (s.e.) associated with the Cox, Extended Cox (ECox) and Aalen models for several scenarios where $X_{1}$ is continuous with time-invariant effect

\begin{tabular}{|c|c|c|c|c|c|c|c|c|c|c|c|c|c|c|}
\hline \multirow[b]{2}{*}{$\%$} & \multirow[b]{2}{*}{$n$} & \multirow[b]{2}{*}{ Model } & \multicolumn{2}{|c|}{$t=2.0$} & \multicolumn{2}{|c|}{$t=3.0$} & \multicolumn{2}{|c|}{$t=3.5$} & \multicolumn{2}{|c|}{$t=4.0$} & \multicolumn{2}{|c|}{$t=4.5$} & \multicolumn{2}{|c|}{$t=5.0$} \\
\hline & & & AUC & s.e. & AUC & s.e. & AUC & s.e. & $\mathrm{AUC}$ & s.e. & AUC & s.e. & AUC & s.e. \\
\hline \multirow{9}{*}{0} & 100 & Cox & 0.955 & 0.020 & 0.946 & 0.022 & 0.944 & 0.023 & 0.940 & 0.026 & 0.933 & 0.027 & 0.934 & 0.033 \\
\hline & 100 & ECox & 0.956 & 0.017 & & 0.020 & 0.945 & 0.023 & 0.941 & 0.028 & - & - & - & - \\
\hline & 100 & Aalen & 0.956 & 0.016 & 0.947 & 0.017 & 0.946 & 0.017 & 0.942 & 0.022 & - & - & - & - \\
\hline & 300 & Cox & 0.957 & 0.017 & 0.948 & 0.018 & 0.944 & 0.018 & 0.941 & 0.020 & 0.941 & 0.020 & 0.939 & 0.022 \\
\hline & 300 & ECox & 0.957 & 0.013 & 0.949 & 0.016 & 0.944 & 0.016 & 0.942 & 0.018 & 0.942 & 0.019 & - & - \\
\hline & 300 & Aalen & 0.958 & 0.008 & 0.950 & 0.011 & 0.946 & 0.011 & 0.943 & 0.013 & 0.942 & 0.013 & - & - \\
\hline & 500 & Cox & 0.959 & 0.013 & 0.948 & 0.013 & 0.949 & 0.013 & 0.947 & 0.016 & 0.945 & 0.017 & 0.939 & 0.020 \\
\hline & 500 & ECox & 0.960 & 0.011 & 0.949 & 0.012 & 0.949 & 0.013 & 0.948 & 0.014 & 0.946 & 0.016 & - & - \\
\hline & 500 & Aalen & 0.961 & 0.006 & 0.951 & 0.008 & 0.949 & 0.008 & 0.949 & 0.010 & 0.947 & 0.012 & - & - \\
\hline \multirow{9}{*}{20} & 100 & Cox & 0.936 & 0.027 & 0.904 & 0.039 & 0.883 & 0.049 & 0.855 & 0.062 & 0.820 & 0.083 & 0.774 & 0.104 \\
\hline & 100 & ECox & 0.936 & 0.027 & 0.903 & 0.040 & 0.882 & 0.049 & 0.854 & 0.062 & - & - & - & - \\
\hline & 100 & Aalen & .938 & 0.030 & 0.885 & 0.049 & 0.842 & 0.097 & 0.796 & 0.06 & - & - & - & - \\
\hline & 300 & Cox & 0 & 0.020 & 0.917 & 0.024 & 0.904 & 0.028 & 0.886 & 0.0 & 0.860 & 0.053 & 0.815 & 0.085 \\
\hline & 300 & ECox & 0.937 & 0.020 & 0.915 & 0.025 & 0.901 & 0.029 & 0.879 & 0.042 & 0.849 & 0.064 & - & - \\
\hline & 300 & Aalen & 0.932 & 0.021 & 0.907 & 0.026 & 0.891 & 0.035 & 0.867 & 0.052 & 0.828 & 0.088 & - & - \\
\hline & 500 & Cox & 0.938 & 0.019 & 0.918 & 0.021 & 0.906 & 0.025 & 0.887 & 0.031 & 0.859 & 0.051 & 0.815 & 0.070 \\
\hline & 500 & ECox & 0.937 & 0.017 & 0.917 & 0.019 & 0.902 & 0.023 & 0.885 & 0.029 & 0.858 & 0.045 & - & - \\
\hline & 500 & Aalen & 0.932 & 0.016 & 0.912 & 0.019 & 0.899 & 0.023 & 0.883 & 0.031 & 0.856 & 0.049 & - & - \\
\hline \multirow{9}{*}{40} & 100 & Cox & 0.905 & 0.039 & 0.852 & 0.058 & 0.818 & 0.071 & 0.779 & 0.081 & 0.734 & 0.094 & 0.686 & 0.109 \\
\hline & 100 & ECox & 0.905 & 0.039 & 0.851 & 0.058 & 0.818 & 0.071 & 0.779 & 0.081 & - & - & - & - \\
\hline & 100 & Aalen & 0.887 & 0.041 & 0.809 & 0.070 & 0.754 & 0.101 & 0.706 & 0.092 & - & - & - & - \\
\hline & 300 & Cox & 0.914 & 0.024 & 0.872 & 0.0 & 0.844 & 0.039 & 0.807 & 0.051 & 0.763 & 0.066 & 0.707 & 0.081 \\
\hline & 300 & ECox & 0.914 & 0.024 & 0.872 & 0.033 & 0.844 & 0.039 & 0.806 & 0.051 & 0.764 & 0.065 & - & - \\
\hline & 300 & Aalen & 0.908 & 0.025 & 0.861 & 0.037 & 0.827 & 0.049 & 0.783 & 0.068 & 0.728 & 0.091 & - & - \\
\hline & 500 & Cox & 0.914 & 0.021 & 0.876 & 0.029 & 0.852 & 0.035 & 0.817 & 0.044 & 0.770 & 0.063 & 0.713 & 0.075 \\
\hline & 500 & ECox & 0.914 & 0.021 & 0.876 & 0.029 & 0.852 & 0.035 & 0.817 & 0.0 & 0.770 & 0.0 & - & - \\
\hline & 500 & Aalen & 0.913 & 0.020 & 0.872 & 0.028 & 0.845 & 0.038 & 0.808 & 0.045 & 0.760 & 0.065 & - & - \\
\hline
\end{tabular}

Note: \% denotes the percentage of censorship and $n$ the sample size.

Table 2 displays the simulation results for those scenarios where a continuous covariate with time-varying effect was considered in the data-generating process (maximum time ranged from 20 to 23 ). The $\operatorname{AUC}(t)$ values for the dynamic models (extended Cox and Aalen models) were restricted to time $t \in(0, \tau]$, with $\tau=13$ for $n=100$, and $\tau=15$ for $n=300$ and 500, respectively. From Table 2, one can observe higher values of $\mathrm{AUC}(t)$ associated with the dynamic models as time increases, showing higher predictive accuracy of these models in relation to the Cox model. As expected, the standard errors decreased as the sample size increased and increased as the percentage of censorship increased. In addition, scenarios with $20 \%$ and $40 \%$ of censorship, when compared with those without censorship, showed a more pronounced decrease in the $\operatorname{AUC}(t)$ values over time. Thus, if the covariate 
has a time-varying effect, the simulation results indicated that the dynamic models, when compared to the Cox model, exhibit higher predictive accuracy.

Table 2 - AUC $(t)$ and standard error (s.e.) associated with the Cox, Extended Cox (ECox) and Aalen models for several scenarios where $X_{1}$ is continuous with time-varying effect

\begin{tabular}{|c|c|c|c|c|c|c|c|c|c|c|c|c|c|c|}
\hline \multirow[b]{2}{*}{$\%$} & \multirow[b]{2}{*}{$n$} & \multirow[b]{2}{*}{ Model } & \multicolumn{2}{|c|}{$t=2.0$} & \multicolumn{2}{|c|}{$t=5.0$} & \multicolumn{2}{|c|}{$t=7.0$} & \multicolumn{2}{|c|}{$t=10.0$} & \multicolumn{2}{|c|}{$t=13.0$} & \multicolumn{2}{|c|}{$t=15.0$} \\
\hline & & & AUC & s.e. & AUC & s.e. & AUC & s.e. & AUC & s.e. & AUC & s.e. & AUC & s.e. \\
\hline \multirow{9}{*}{0} & 100 & Cox & 0.981 & & 0.960 & & 0.942 & 0.017 & 0.915 & & 0.908 & 0.043 & 0.904 & 0.052 \\
\hline & 100 & & & & 974 & & & & 934 & & & & - & - \\
\hline & 100 & & & & & & & & & & & & - & - \\
\hline & 300 & & & & & & & & & & & & 0.915 & 0.023 \\
\hline & 300 & & & & & & & & & & & & & \\
\hline & & & & & & & & & & & & & & 0.026 \\
\hline & & & & & & & & & & & & & & .021 \\
\hline & & & & & & & & & & & & & & .021 \\
\hline & & & & & & & & & & & & & .937 & 0.021 \\
\hline \multirow{9}{*}{20} & & & & & & & & & & & & & 0.760 & 0.064 \\
\hline & & & & & & & & & & & & & - & - \\
\hline & 100 & & & & & & & & & & & & - & - \\
\hline & 300 & & & & & & & & & & & & & 0.058 \\
\hline & 300 & & & & & & & & & & & & & 0.051 \\
\hline & 300 & & & & & & & & & & & & & 0.048 \\
\hline & 500 & & & & & & & & & & & & & 0.043 \\
\hline & & & & & & & & & & & & & & 0.042 \\
\hline & & & & & & & & & .892 & & & & 0.827 & 0.040 \\
\hline \multirow{9}{*}{40} & & & & & & & & & & & & & 0.591 & 0.091 \\
\hline & & & & & & & & & & & & & - & - \\
\hline & & & & & & & & & & & & & - & - \\
\hline & & & & & & & & & & & & & & \\
\hline & 300 & & & & & & & & & & & & .703 & 0.061 \\
\hline & 300 & & & & & & & & & & & & .706 & 0.060 \\
\hline & 500 & & & & & & & & & & & & 0.676 & 0.045 \\
\hline & & & & & & & & & & & & & 0.723 & 0.054 \\
\hline & 500 & Aalen & 0.974 & 0.009 & 0.935 & 0.015 & 0.899 & 0.018 & 0.839 & 0.028 & 0.762 & 0.031 & 0.726 & 0.053 \\
\hline
\end{tabular}

Note: \% denotes the percentage of censorship and $n$ the sample size.

Simulations were also carried out considering the covariate $X_{1}$ as dichotomous (with time-invariant effect and time-varying effect). From the results (not shown), it was possible to obtain the same conclusions presented for $X_{1}$ continuous. However, although the $\mathrm{AUC}(t)$ values for $X_{1}$ dichotomous have presented the same behavior observed for $X_{1}$ continuous, the magnitude of the $\mathrm{AUC}(t)$ values was somewhat lower for $X_{1}$ considered as dichotomous rather than continuous (probably because dichotomous covariates are usually less informative than continuous ones).

In the next section, the standard Cox model and the dynamic survival models mentioned in this paper are considered for analysing the PBC data. The main purpose of this analysis is to illustrate the results obtained in the simulation study by means of a real situation which has several covariates.

\section{Illustration}

The standard Cox model and all other hazards models mentioned in this paper were considered for analysing the PBC data (FLEMING; HARRINGTON, 1991). This dataset refers to a study conducted at the Mayo Clinic between 1974 and 
1984 which involved 418 patients diagnosed with primary biliary cirrhosis (PBC) of the liver. They were followed until death or censoring. In addition to time at risk (in years) and censoring indicator, five covariates were considered in the analysis: age (in years), edema (present or absent), serum albumin ( $\mathrm{g} / \mathrm{dl}$ ), serum bilirubin $(\mathrm{mg} / \mathrm{dl})$ and prothrombin time (in seconds). A summary of them is shown in Table 3. The last three covariates were used on the logarithmic scale and all continuous covariates were centered around their respective averages. At the end of the study it was recorded around $55 \%$ of censorship.

Table 3 - Covariates considered in the analysis of the PBC dataset

\begin{tabular}{ll}
\hline Covariates & Summary \\
\hline Age & 26 to 78 year $($ mean $=51 ;$ s.d. $=10.47)$ \\
Edema & 352 absent $(85 \%)$ \\
Serum bilirubin & 0.3 to $28 \mathrm{mg} / \mathrm{dl}($ mean $=3.2 ;$ s.d. $=4.38)$ \\
Serum albumin & 1.96 to $4.64 \mathrm{mg} / \mathrm{dl}($ mean $=3.5 ;$ s.d. $=0.42)$ \\
Prothrombin time & 9 to 18 seconds $($ mean $=10.73 ;$ s.d. $=1.02)$ \\
\hline
\end{tabular}

Initially, models (1), (2) and (4) were fitted including all covariates. Next, graphical methods (not shown) and tests were carried out to assess the significance of the covariate effects (that is, to test the null hypothesis $H_{01}: \beta_{k}(t)=0$ ), as well as the validity of the proportional hazards assumption, $H_{02}: \beta_{k}(t)=\beta_{k}, k=1, \ldots, 5$. Wald test and a test based on Schoenfeld residuals were used, respectively, to test the null hypotheses for the Cox model while supremum tests by Martinussen and Scheike (2006) were used for the other two models. Estimation for models with time-varying covariate effects was restricted to the time interval $(0,8.1]$, since $\tau=8.1$ was the maximum time at which the design matrix $\mathbf{X}(t)$ was non-singular.

From the p-values displayed in Table 4 related to the tests performed, it can be seen that all covariates showed significant effect and that the proportional hazards assumption was not satisfied for two covariates: edema and $\log$ (prothrombin). Thus, models (3) and (5) were fitted since some covariates have revealed significant timevarying effect and others do not.

Table 4 - P-values from tests used to assess the significance of the covariate effects $\left(H_{01}^{*}: \beta_{k}=0\right.$ or $\left.H_{01}: \beta_{k}(t)=0\right)$ and the proportionality $\left(H_{02}: \beta_{k}(t)=\beta_{k}\right)$

\begin{tabular}{|c|c|c|c|c|c|c|}
\hline \multirow[b]{2}{*}{ Covariates } & \multicolumn{2}{|c|}{$\begin{array}{l}\text { Standard } \\
\text { Cox model }\end{array}$} & \multicolumn{2}{|c|}{$\begin{array}{l}\text { Extended } \\
\text { Cox model }\end{array}$} & \multicolumn{2}{|c|}{ Aalen model } \\
\hline & $H_{01}^{*}$ & $H_{02}$ & $H_{01}$ & $H_{02}$ & $H_{01}$ & $H_{02}$ \\
\hline age & 0.001 & 0.374 & 0.001 & 0.612 & 0.080 & 0.970 \\
\hline edema & 0.008 & 0.038 & 0.002 & $<0.001$ & 0.009 & $<0.001$ \\
\hline $\log ($ albumin $)$ & $<0.001$ & 0.277 & $<0.001$ & 0.921 & 0.002 & 0.959 \\
\hline $\log$ (bilirubin) & $<0.001$ & 0.218 & $<0.001$ & 0.740 & $<0.001$ & 0.272 \\
\hline $\log$ (prothrombin) & 0.013 & $<0.001$ & $<0.001$ & 0.002 & 0.008 & 0.001 \\
\hline
\end{tabular}


In order to evaluate the predictive accuracy of models (1), (3) and (5), the $\mathrm{AUC}(t)$ was calculated at several times. Bootstrap resampling (EFRON, 1982) was considered for assessing the standard errors of the $\mathrm{AUC}(t)$. The results are shown in Table 5 and depicted in Figure 2(a). The decrease observed in the $\mathrm{AUC}(t)$ estimates is expected since there is a gradual decrease in the set of subjects at risk over time.

From Table 5 and Figure 2(a), it can be noted that models (3) and (5) showed better predictive accuracy than the Cox model (1). This result is consistent with what was expected since the proportional hazards assumption was not satisfied for two of the five covariates in the models. In addition, it can be noted that models (3) and (5) provided similar predictive accuracy over time. It shows that even belonging to distinct frameworks (model (3) is multiplicative and model (5) is additive), these two models have provided very close prediction of those subjects who will or will not experience the event at time $t$.

Table 5 - AUC $(t)$ estimates and respective standard errors

\begin{tabular}{|c|c|c|c|}
\hline $\operatorname{AUC}(t)$ & $\begin{array}{c}\text { Model (1) } \\
\text { Standard Cox } \\
\text { NNE (s.e.) }\end{array}$ & $\begin{array}{c}\text { Model (3) } \\
\text { Multiplicative } \\
\text { NNE (s.e.) }\end{array}$ & $\begin{array}{c}\text { Model (5) } \\
\text { Additive } \\
\text { NNE (s.e.) }\end{array}$ \\
\hline$t=0.5$ & $0.924(0.031)$ & $0.919(0.035)$ & $0.932(0.028)$ \\
\hline$t=1.5$ & $0.851(0.038)$ & $0.883(0.029)$ & $0.883(0.031)$ \\
\hline$t=3.0$ & $0.848(0.022)$ & $0.870(0.021)$ & $0.871(0.021)$ \\
\hline$t=5.0$ & $0.841(0.023)$ & $0.875(0.019)$ & $0.872(0.018)$ \\
\hline$t=7.0$ & $0.818(0.023)$ & $0.857(0.027)$ & $0.843(0.020)$ \\
\hline$t=8.0$ & $0.792(0.027)$ & $0.819(0.034)$ & $0.828(0.025)$ \\
\hline
\end{tabular}

Next, to assess and compare the overall goodness-of-fit of models (1), (3) and (5), the Cox-Snell residuals $e_{i}$ against the cumulative hazard rate $\widehat{\Lambda}\left(e_{i}\right)$ were plotted in Figure 2(b). Inspection of the curves depicted in this figure suggests the models with time-varying covariate effects (models (3) and (5)) as those fitting best the data. Hence, it could be reasonable to consider these two models instead of choosing one in order to obtain complementary information from each other since the additive model provides information on the risk in absolute terms while the multiplicative model in relative terms. Anyway, to choose a model under a particular framework, it can be advisable to taken into account the objectives of the study, facilities of estimation and interpretation, sample size, number of subjects at risk at time $t$, percentage of censorship, and amount of covariates of interest, amongst others.

\section{Discussion}

The additive and multiplicative hazards models postulate distinct relationships between the hazard and covariates. Often, it is unclear which of these models should be preferred in a specific application. The additive Aalen's model can naturally model changing covariate effects. However, estimation of the regression functions in this model is only applicable while the design matrix $\mathbf{X}(t)$ is non-singular. 
(a)

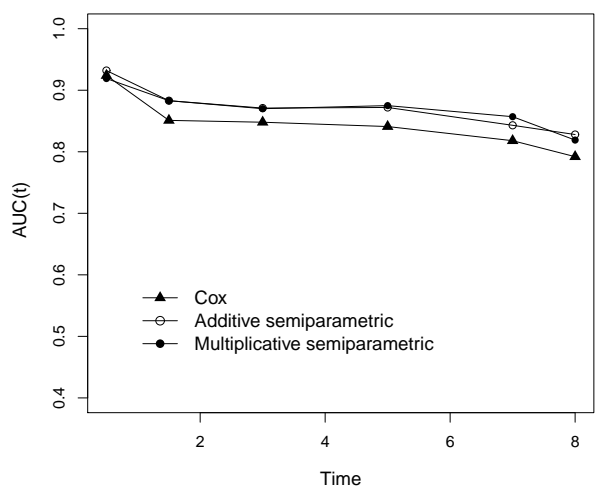

(b)

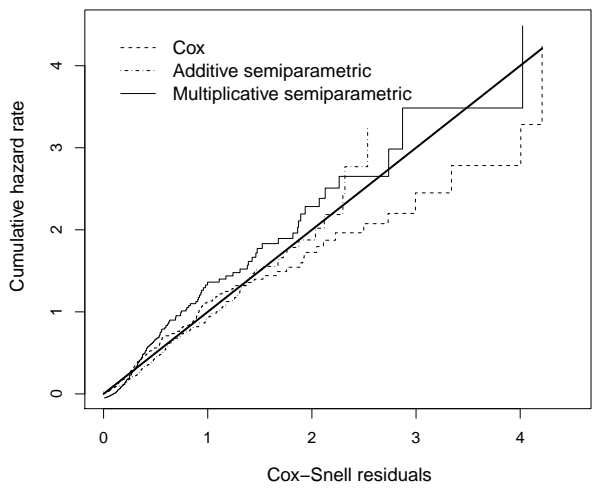

Figure 2 - (a) Time $t$ against $\mathrm{AUC}(t)$ and (b) Cox-Snell residuals $e_{i}$ against the estimated cumulative hazard rate $\widehat{\Lambda}\left(e_{i}\right)$ for models (1), (3) and (5).

As mentioned by Zhang and Akcin (2012), this requirement has undesirable consequences, particularly when there are many covariates in the sample. For instance, from at a specific time $t$ of the study period, the reduced set of subjects at risk may not yield a design matrix with full rank. Hence, the regression functions are inestimable even though the failures are still observed. In addition, for small to medium sized data the Aalen's model and the extended Cox model with all covariate effects being time-varying may further be difficult to fit. Hence, Martinussen and Scheike (2006) suggest that semiparametric models can provide a more reasonable compromise between model complexity and size of the data.

Parameter estimation in hazards models with time-varying covariate effects is more complex than in the Cox model, being highly impacted due to the sample size, number of subjects at risk at time $t$, and percentage of censorship. Thus, although these models are flexible for allowing covariates with time-varying effect, the estimation procedures commonly used to estimate a such effect are restricted to a time $t$ usually smaller than the maximum time observed in the data set, making the mentioned flexibility less favorable in practice than would be expected. However, even when the models with time-varying covariate effects provide themselves as viable options, it is important to point out the relevance of evaluating how serious is the violation of the proportional hazards assumption. If such violation is not too serious, it may be more appropriate models with no time-varying covariate effects.

From the Mayo PBC data analysis, in which the $\mathrm{AUC}(t)$ was considered as a procedure for helping us in the comparison of hazards models under distinct frameworks, it was possible to observe that all models have provided similar results regarding covariates selected to remain in the final model. All models have also indicated the same set of covariates with time-varying effect. As expected in this case, the $\mathrm{AUC}(t)$ showed better predictive accuracy associated with the models that allow some covariates with time-varying effect and others do not. The $\operatorname{AUC}(t)$ has 
also indicated that hazards models under distinct frameworks can provide similar predictive accuracy. This result is consistent with the conclusion provided by some authors (TORNER, 2004; CAO, 2005; ABADI et al., 2011) that multiplicative and additive hazards models should be seen as complementary methods that can together provide a fuller understanding of the data.

From all that was presented and discussed, the graphical representation based on the Cox-Snell residuals together with the time-dependent version of the area under the ROC curve, $\operatorname{AUC}(t)$, seem to be useful procedures that can help us to compare hazards models formulated under distinct frameworks regarding to their overall goodness-of-fit and predictive accuracy. Drawing a parallel with other measures, such as the Akaike's information criterion (AIC), the AUC $(t)$ could be viewed as a measure that can help us to compare models that are or not nested, as well as to compare nonparametric, semiparametric and parametric hazards models amongst them in order to assist us in choosing those that best fit the data.

\section{Acknowledgments}

We would like to thank editors and reviewers for their comments and suggestions.

RAMINELLI, J. A.; GIOLO, S. R. Modelos de sobrevivência no contexto de riscos multiplicativos e aditivos. Rev. Bras. Biom., Lavras, v.37, n.3, p.306-323, 2019.

- RESUMO: Em análise de sobrevivência, os modelos de riscos multiplicativos e aditivos fornecem as duas principais abordagens para estudar a associação entre o risco e as covariáveis. Quando esses modelos são considerados para analisar certo conjunto de dados de sobrevivência, torna-se importante avaliar a qualidade de ajuste dos modelos e o quão bem cada um deles pode predizer aqueles indivíduos que subsequentemente irão ou não experimentar o evento. Nesse artigo, essa avaliação é feita com base em uma representação gráfica dos resíduos de Cox-Snell e, também, em um versão tempodependente da área sob a curva ROC (receiver operating characteristic), denotada por $A U C(t)$. Um estudo de simulação é realizado para avaliar a performance da $A U C(t)$ como uma ferramenta de comparação do poder preditivo de modelos de sobrevivência. Um conjunto de dados da Mayo Clinic sobre cirrose biliar primária $(P B C)$ do fígado é considerado a fim de ilustrar a utilidade dessas ferramentas para a comparação de modelos de sobrevivência formulados sob estruturas de riscos distintas.

- PALAVRAS-CHAVE: Comparação de modelos; ROC; tempos de vida; poder preditivo.

\section{References}

AALEN, O. O. A model for non-parametric regression analysis of counting processes. In: KLONECKI, W.; KOZEK, A.; ROSINSKI, J. (Eds.). Mathematical 
Statistics and Probability Theory: Lecture Notes in Statistics. New York: SpringerVerlag, v.2, 1980.

AALEN, O. O. A linear regression model for the analysis of lifetime. Statistics in Medicine, v.8, p.907-925, 1989.

ABADI, A.; SAADAT, S.; YAVARI, P.; BAJDIK, C.; JALILI, P. Comparison of Aalen's additive and Cox proportional hazards models for breast cancer survival: analysis of population-based data from British Columbia, Canada. Asian Pacific Journal of Cancer Prevention, v.12, p.3113-3116, 2011.

AKRITAS, M. G. Nearest neighbor estimation of a bivariate distribution under random censoring. Annals of Statistics, v.22, p.1299-1327, 1994.

AUSTIN, P. C. Generating survival times to simulate Cox proportional hazards models with time-varying covariates. Statistics in Medicine. v.31, p.3946-3958, 2012.

BENDER, R.; AUGUSTIN, T; BLETTNER, M. Generating survival times to simulate Cox proportional hazards models. Statistics in Medicine. v.24, p.1713-1723, 2005.

BLANCHE, P.; DARTIGUES, J. F.; JACQMIN-GADDA, H. Review and comparison of ROC curve estimators for a time-dependent outcome with markerdependent censoring. Biometrical Journal, v.55, n.5, p.687-704, 2013.

CAI, Z.; SUN, Y. Local linear estimation for time-dependent coefficients in Cox's regression models. Scandinavian Journal of Statistics, v.30, p.93-112, 2003.

$\mathrm{CAO}, \mathrm{H}$. A comparison between the additive and multiplicative risk models. Master's Dissertation. Faculté des Sciences, Génie Université Laval, Québec, 2005.

CHEN, K.; LIN, H.; ZHOU, Y. Efficient estimation for the Cox model with varying coefficients. Biometrika, v.99, p.379-392, 2012.

COX, D. R. Regression models and life tables (with discussion). Journal of the Royal Statistical Society B, v.34, p.187-220, 1972.

COX, D. R. Partial likelihood. Biometrika, v.62, p.269-276, 1975.

COX, D. R., SNELL, E. J. A general definition of residuals. Journal of the Royal Statistical Society B, v.30, p.248-275, 1968.

EFRON, B. The jackknife, the bootstrap, and other resampling plans. Philadelphia: Society for Industrial and Applied Mathematics (SIAM), 1982.

FLEMING, T. R.; HARRINGTON, D. P. Counting processes and survival analysis. New York: John Wiley \& Sons, 1991.

GRAMBSCH, P. M.; THERNEAU, T. M. Proportional hazards tests and diagnostics based on weighted residuals. Biometrics, v.81, p.515-526, 1994.

Rev. Bras. Biom., Lavras, v.37, n.3, p.306-323, 2019 - doi: 10.28951/rbb.v37i3.391 
HASTIE, T.; TIBSHIRANI, R.; FRIEDMAN, J. The elements of statistical learning. New York: Springer-Verlag, 2009.

HEAGERTY, P. J.; LUMLEY, T.; PEPE, M. S. Time-dependent ROC curves for censored survival data and a diagnostic marker. Biometrics, v.56, p.337-344, 2000.

HEAGERTY, P. J.; ZHENG, Y. Survival model predictive accuracy and ROC curves. Biometrics, v.61, p.92-105, 2005.

HUFFER, F. W.; McKEAGUE, I. W. Weighted least squares estimation for Aalen's additive risk model. Journal of the American Statistical Association, v.86, p.114-29, 1991.

KAMARUDIN, A. N.; COX, T.; KOLAMUNnAGE-DONA, R. Time-dependent ROC curve analysis in medical research: current methods and applications. BMC Medical Research Methodology, v.17, p.53-481, 2017. Open access. Available at: https://bmcmedresmethodol.biomedcentral.com/track/pdf/10.1186/s12874017-0332-6 (Accessed: 07 June 2018).

LAWLESS, J. Statistical models and methods for lifetime data. New York: John Wiley \& Sons, 2002.

LIN, D. Y.; WEI, L. J.; YING, Z. Checking the Cox model with cumulative sums of martingale-based residuals. Biometrika, v.80, p.557-572, 1993.

MARTINUSSEN, T.; SCHEIKE, T. H. Dynamic regression models for survival data. New York: Springer-Verlag, 2006.

MARTINUSSEN, T.; SCHEIKE, T. H.; SKOVGAARD, I. M. Efficient estimation of fixed and time-varying covariate effects in multiplicative intensity models. Scandinavian Journal of Statistics, v.29, p.57-74, 2002.

McKEAGUE, I. W.; SASIENI, P. D. A partly parametric additive risk model. Biometrika, v.81, p.501-514, 1994.

MURPHY, S. A.; SEN, P. K. Time-dependent coefficients in a Cox-type regression model. Stochastic Processes and Their Applications, v.39, p.153-180, 1991.

R CORE TEAM. R: A language and environment for statistical computing. Vienna, Austria, 2017. Available at: http://www.R-project.org/ (Accessed: 17 June 2017).

SCHEIKE, T. H. The additive nonparametric and semiparametric Aalen model as the rate function for a counting process. Lifetime Data Analysis, v.8, p.247-262, 2002 .

SCHOENFELD, D. Partial residuals for the proportional hazard regression model. Biometrika, v.69, p.239-241, 1982.

SHEN, W.; NING, J.; YUAN, Y. A direct method to evaluate the time-dependent predictive accuracy for biomarkers. Biometrics, v.71, n.2, p.439-449, 2015. 
TORNER, A. Proportional hazards and additive regression analysis of survival for severe breast cancer. Stockholm University. Technical Report, 2004. Available online at: http://www2. math.su.se/matstat/reports/serieb/2004/rep3/report.pdf (Accessed: 10 May 2017).

ZHANG, X.; AKCIN, H. (2012) A SAS macro for direct adjusted survival curves based on Aalen's additive model. Computer Methods and Programs in Biomedicine, v.108, p. 310-317, 2012.

ZUCKER, D. M., KARR, A. F. Nonparametric survival analysis with timedependent covariate effects: A penalized partial likelihood approach. The Annals of Statistics, v.18, p.329-353, 1990.

Received on 13.08.2018.

Approved after revised on 03.05.2019. 


\section{APPENDIX A - R commands used to obtain the AUC $(t)$}

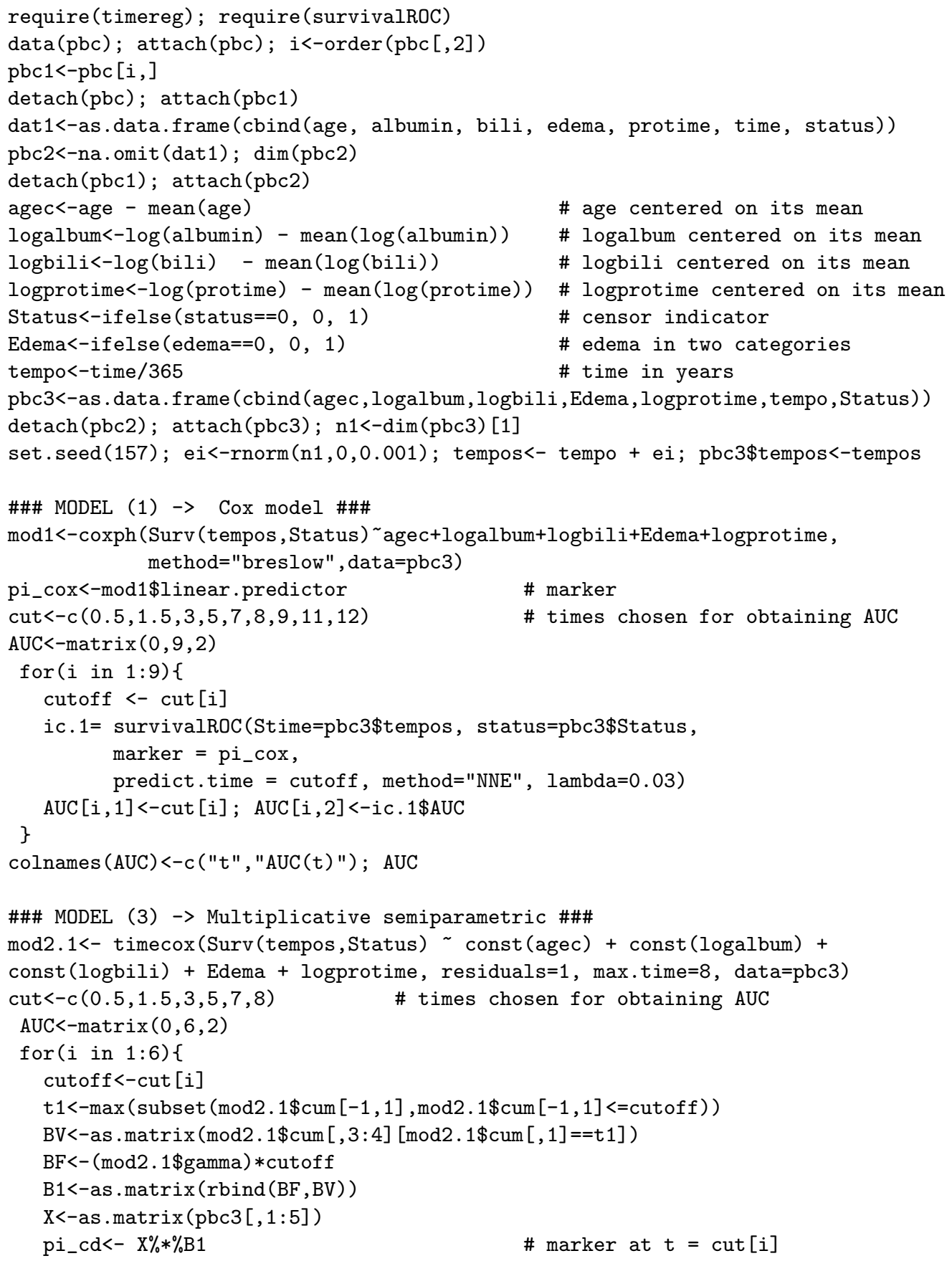




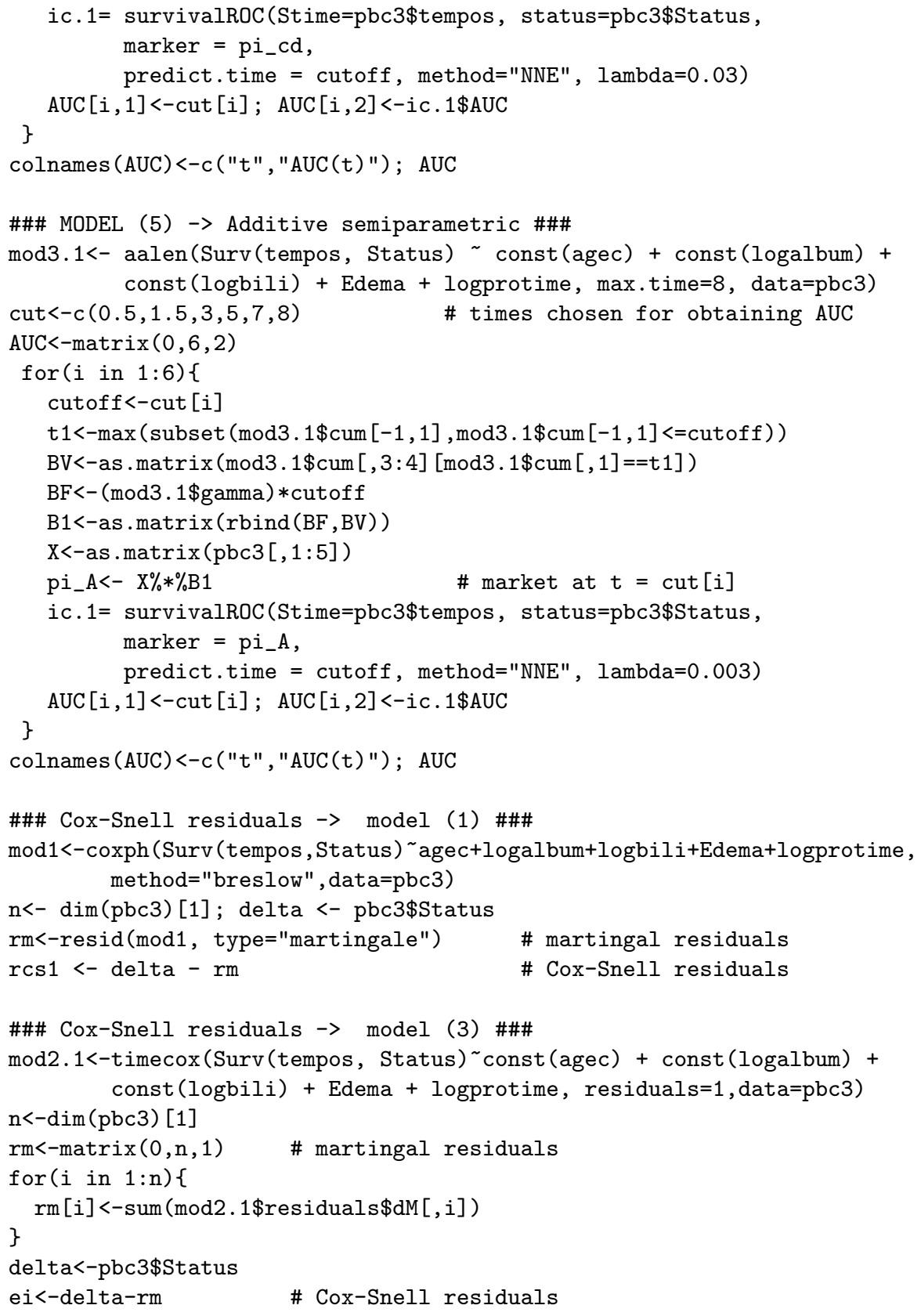

